\title{
An isoperimetric problem for point interactions
}

\author{
Pavel Exner \\ Department of Theoretical Physics, Nuclear Physics Institute, \\ Academy of Sciences, 25068 Rež near Prague, Czechia, and \\ Doppler Institute, Czech Technical University, Břehová 7, \\ 11519 Prague, Czechia \\ exner@ujf.cas.cz
}

\begin{abstract}
We consider Hamiltonian with $N$ point interactions in $\mathbb{R}^{d}, d=2,3$, all with the same coupling constant, placed at vertices of an equilateral polygon $\mathcal{P}_{N}$. It is shown that the ground state energy is locally maximized by a regular polygon. The question whether the maximum is global is reduced to an interesting geometric problem.
\end{abstract}

\section{Introduction}

Questions about geometrical configurations which lead to extremal value of a spectral quantity represent a classical topic in mathematical physics; recall the Faber-Krahn inequality [Fa, $\mathrm{Kr}$, the PPW-conjecture proved by Ashbaugh and Benguria $\mathrm{AB}$, and numerous other examples. A particular place in this list is occupied by the Dirichlet problem for the Laplacian in annular strips and their higher-dimensional analogues where the principal eigenvalue is typically maximized by a circular shape EHL.

The reason behind the last named result is an effective attraction coming from the curvature. This effect is robust and can be manifested in situation where the confinement to the vicinity of a certain geometric object is much weaker than boundary conditions, being realized, for instance, by a potential or even by a mere family of point interactions. An illustration is is provided 
by "polymer" models [AGHH], i.e. infinite equidistant arrays of point interactions: if such a polymer is curved but asymptotically straight in a suitable sense, it has a nonempty discrete spectrum the properties of which depend substantially on the geometry of the array [Ex1, EN].

It is natural to ask whether the mentioned results about Dirichlet annuli have an analogue in the situation when the point interactions are arranged along a closed curve of a fixed length. In this paper we address this isoperimetric problem and show that the "circular" shape, namely a regular polygon is a local maximizer for the lowest eigenvalue.

On the other hand, the question about the global uniqueness of this maximizer is left open. As we shall see in Sec. 3 below, the problem can be reduced to verification of a simple property for some families of polygon diagonals. At a glance it seems to be something which must be known since Euclid's Elementa, or at least for quite a long time. However, this impression is wrong; it is found nowhere in the literature unless I looked the wrong direction and asked wrong people. And as any problem which allow a statement in elementary geometric terms, it has a certain independent appeal.

We will formulate the problem and state our main result, Theorem 2.1. in the next section. It will be then proved in the Secs 3 and 4 , while the last two sections are devoted to the global uniqueness question and possible extensions of the result.

\section{The main result}

Let $\mathcal{P}_{N} \subset \mathbb{R}^{d}, d=2,3$, be a polygon which it is for the present purpose convenient to identify with an ordered set of its vertices, $\mathcal{P}_{N}=\left\{y_{1}, \ldots, y_{N}\right\}$; if the vertex indices exceed this range they are understood $\bmod N$. We suppose that $\mathcal{P}_{N}$ is equilateral, $\left|y_{i+1}-y_{i}\right|=\ell$ for a fixed $\ell>0$ and any $i$. By $\tilde{\mathcal{P}}_{N}$ we denote a regular polygon of the edge length $\ell$, which means planar (this is trivial if $d=2$ ) with vertices lying on a circle of radius $\ell\left(2 \sin \frac{\pi}{N}\right)^{-1}$.

The object of our interest is the Hamiltonian $-\Delta_{\alpha, \mathcal{P}_{N}}$ in $L^{2}\left(\mathbb{R}^{d}\right)$ with $N$ point interactions, all of the same coupling constant $\alpha$, placed at the vertices of $\mathcal{P}_{N}$. We suppose that this operator has a non-empty discrete spectrum,

$$
\epsilon_{1} \equiv \epsilon_{1}\left(\alpha, \mathcal{P}_{N}\right):=\inf \sigma\left(-\Delta_{\alpha, \mathcal{P}_{N}}\right)<0,
$$

which is satisfied for any $\alpha \in \mathbb{R}$ if $d=2$, while in the case $d=3$ it is true below a certain critical value of $\alpha-$ cf. AGHH, Sec. II.1]. 
Theorem 2.1 Under the stated conditions, $\epsilon_{1}\left(\alpha, \mathcal{P}_{N}\right)$ is for fixed $\alpha$ and $\ell$ locally sharply maximized by a regular polygon, $\mathcal{P}_{N}=\tilde{\mathcal{P}}_{N}$.

Let us remark that speaking about uniqueness of the maximizer, we have of course in mind the family of regular polynomials related mutually by Euclidean transformations of $\mathbb{R}^{d}$.

\section{A geometric reformulation}

As the first step to prove Theorem 2.1] we want to show that the task can be reduced to a geometric problem. Using the standard notation, $k=i \kappa$ with $\kappa>0$, we find the eigenvalues $-\kappa^{2}$ solving the following spectral condition,

$$
\operatorname{det} \Gamma_{k}=0 \quad \text { with } \quad\left(\Gamma_{k}\right)_{i j}:=\left(\alpha-\xi^{k}\right) \delta_{i j}-\left(1-\delta_{i j}\right) g_{i j}^{k},
$$

where $g_{i j}^{k}:=G_{k}\left(y_{i}-y_{j}\right)$, or equivalently

$$
g_{i j}^{k}=\left\{\begin{array}{ccc}
\frac{1}{2 \pi} K_{0}\left(\kappa\left|y_{i}-y_{j}\right|\right) & \ldots & d=2 \\
\frac{e^{-\kappa\left|y_{i}-y_{j}\right|}}{4 \pi\left|y_{i}-y_{j}\right|} & \ldots & d=3
\end{array}\right.
$$

and the regularized Green's function at the interaction site is

$$
\xi^{k}=\left\{\begin{array}{ccc}
-\frac{1}{2 \pi}\left(\ln \frac{\kappa}{2}+\gamma_{\mathrm{E}}\right) & \ldots & d=2 \\
-\frac{\kappa}{4 \pi} & \ldots & d=3
\end{array}\right.
$$

The matrix $\Gamma_{i \kappa}$ has $N$ eigenvalues counting multiplicity which are decreasing in $(-\infty, 0)$ as functions of $\kappa$ - see $[\mathrm{KL}$ and recall that they are real-analytic and non-constant in view of their known asymptotic behavior $\mathrm{AGHH}$. The quantity in question, $\epsilon_{1}\left(\alpha, \mathcal{P}_{N}\right)$, corresponds to the point $\kappa$ where the lowest of these eigenvalues vanishes. Consequently, we have to check that

$$
\min \sigma\left(\Gamma_{i \tilde{\kappa}_{1}}\right)<\min \sigma\left(\tilde{\Gamma}_{i \tilde{\kappa}_{1}}\right)
$$

holds locally for $\mathcal{P}_{N} \neq \tilde{\mathcal{P}}_{N}$, where $-\tilde{\kappa}_{1}^{2}=\epsilon_{1}\left(\alpha, \tilde{\mathcal{P}}_{N}\right)$.

Next we notice that the lowest eigenvalue of $\tilde{\Gamma}_{i \tilde{\kappa}_{1}}$ corresponds to the eigenvector $\tilde{\phi}_{1}=N^{-1 / 2}(1, \ldots, 1)$. Indeed, by [AGHH] there is a bijective correspondence between an eigenfunction $c=\left(c_{1}, \ldots, c_{N}\right)$ of $\Gamma_{i \kappa}$ at the point, where the corresponding eigenvalue equals zero, and the corresponding eigenfunction of $-\Delta_{\alpha, \mathcal{P}_{N}}$ given by $c \leftrightarrow \sum_{j=1}^{N} c_{j} G_{i \kappa}\left(\cdot-y_{j}\right)$, up to a normalization. 
Again by $\mathrm{AGHH}$, the principal eigenvalue of $-\Delta_{\alpha, \mathcal{P}_{N}}$ is simple, so it has to be associated with a one-dimensional representation of the corresponding discrete symmetry group of $\tilde{\mathcal{P}}_{N}$; it follows that $c_{1}=\ldots=c_{N}$. Hence

$$
\min \sigma\left(\tilde{\Gamma}_{i \tilde{\kappa}_{1}}\right)=\left(\tilde{\phi}_{1}, \tilde{\Gamma}_{i \tilde{\kappa}_{1}} \tilde{\phi}_{1}\right)=\alpha-\xi^{i \tilde{\kappa}_{1}}-\frac{2}{N} \sum_{i<j} \tilde{g}_{i j}^{i \tilde{\kappa}_{1}}
$$

On the other hand, for the 1.h.s. of (3.2) we have a variational estimate

$$
\min \sigma\left(\Gamma_{i \tilde{\kappa}_{1}}\right) \leq\left(\tilde{\phi}_{1}, \Gamma_{i \tilde{\kappa}_{1}} \tilde{\phi}_{1}\right)=\alpha-\xi^{i \tilde{\kappa}_{1}}-\frac{2}{N} \sum_{i<j} g_{i j}^{i \tilde{\kappa}_{1}}
$$

and therefore it is sufficient to check that the inequality

$$
\sum_{i<j} G_{i \kappa}\left(y_{i}-y_{j}\right)>\sum_{i<j} G_{i \kappa}\left(\tilde{y}_{i}-\tilde{y}_{j}\right)
$$

holds for all $\kappa>0$ and $\mathcal{P}_{N} \neq \tilde{\mathcal{P}}_{N}$ in the vicinity of the regular polygon $\tilde{\mathcal{P}}_{N}$.

For brevity we introduce the symbol $\ell_{i j}$ for the diagonal length $\left|y_{i}-y_{j}\right|$ and $\tilde{\ell}_{i j}:=\left|\tilde{y}_{i}-\tilde{y}_{j}\right|$. We define the function $F:\left(\mathbb{R}_{+}\right)^{N(N-3) / 2} \rightarrow \mathbb{R}$ by

$$
F\left(\left\{\ell_{i j}\right\}\right):=\sum_{m=2}^{[N / 2]} \sum_{|i-j|=m}\left[G_{i \kappa}\left(\ell_{i j}\right)-G_{i \kappa}\left(\tilde{\ell}_{i j}\right)\right]
$$

notice that $m=1$ does not contribute due to the assumed equilaterality of $\mathcal{P}_{N}$. Our aim is to show that $F\left(\left\{\ell_{i j}\right\}\right)>0$ except if $\left\{\ell_{i j}\right\}=\left\{\tilde{\ell}_{i j}\right\}$. We use the fact that the function $G_{i \kappa}(\cdot)$ is convex for any fixed $\kappa>0$ and $d=2,3$ as it can be seen from cf. (3.1); this yields the inequality

$$
F\left(\left\{\ell_{i j}\right\}\right) \geq \sum_{m=2}^{[N / 2]} \nu_{m}\left[G_{i \kappa}\left(\frac{1}{\nu_{m}} \sum_{|i-j|=m} \ell_{i j}\right)-G_{i \kappa}\left(\tilde{\ell}_{1,1+m}\right)\right]
$$

where $\nu_{n}$ is the number of the appropriate diagonals,

$$
\nu_{m}:=\left\{\begin{array}{ccc}
N & \ldots & m=1, \ldots,\left[\frac{1}{2}(N-1)\right] \\
\frac{1}{2} N & \ldots & m=\frac{1}{2} N \quad \text { for } N \text { even }
\end{array}\right.
$$


At the same time, $G_{i \kappa}(\cdot)$ is monotonously decreasing in $(0, \infty)$, so the sought claim would follow if we demonstrate the inequality

$$
\tilde{\ell}_{1, m+1} \geq \frac{1}{\nu_{n}} \sum_{|i-j|=m} \ell_{i j}
$$

and show that it is sharp for at least one value of $m$ if $\mathcal{P}_{N} \neq \tilde{\mathcal{P}}_{N}$.

Thus we have managed to reformulate our problem in purely geometric terms. Since the corresponding property - to be checked in the following - may be of independent interest we will state it more generally, without dimensional restrictions. Let $\mathcal{P}_{N}$ be an equilateral polygon in $\mathbb{R}^{d}, d \geq 2$. Given a fixed integer $m=2, \ldots,\left[\frac{1}{2} N\right]$ we denote by $\mathcal{D}_{m}$ the sum of lengths of all $m$-diagonals, i.e. the diagonals jumping over $m$ vertices.

$\left(P_{m}\right)$ The quantity $\mathcal{D}_{m}$ is, in the set of equilateral polygons $\mathcal{P}_{N} \subset \mathbb{R}^{d}$ with a fixed edge length $\ell>0$, uniquely maximized by $\tilde{\mathcal{D}}_{m}$ referring to the (family of) regular polygon(s) $\tilde{\mathcal{P}}_{N}$.

\section{A local maximizer}

Our next goal is to demonstrate the following claim which yields in the particular cases $d=2,3$ our main result, Theorem 2.1.

Theorem 4.1 The property $\left(P_{m}\right)$ holds locally for any $m=2, \ldots,\left[\frac{1}{2} N\right]$.

Proof: We have to find, for instance, local maxima of the function

$$
f_{m}: f_{m}\left(y_{1}, \ldots, y_{N}\right)=\frac{1}{N} \sum_{i=1}^{N}\left|y_{i}-y_{i+m}\right|
$$

under the constraints $g_{i}\left(y_{1}, \ldots, y_{n}\right)=0$, where

$$
g_{i}\left(y_{1}, \ldots, y_{n}\right):=\ell-\left|y_{i}-y_{i+1}\right|, \quad i=1, \ldots, N .
$$

The number of independent variables is in fact $(N-2)(d-1)-1$ because $2 d-1$ parameters are related to Euclidean transformations and can be fixed. We put

$$
K_{m}\left(y_{1}, \ldots, y_{N}\right):=f_{m}\left(y_{1}, \ldots, y_{N}\right)+\sum_{r=1}^{N} \lambda_{r} g_{r}\left(y_{1}, \ldots, y_{n}\right)
$$


and compute the derivatives $\nabla_{j} K_{m}\left(y_{1}, \ldots, y_{N}\right)$ which are equal to

$$
\frac{1}{N}\left\{\frac{y_{j}-y_{j+m}}{\left|y_{j}-y_{j+m}\right|}+\frac{y_{j}-y_{j-m}}{\left|y_{j}-y_{j-m}\right|}\right\}-\lambda_{j} \frac{y_{j}-y_{j+1}}{\ell}-\lambda_{j-1} \frac{y_{j}-y_{j-1}}{\ell} .
$$

We want to show that these expressions vanish for a regular polygon. Let us introduce a parametrization for any planar equilateral polygon. Without loss of generality we may suppose that it lies in the plane of the first two axes. The other coordinates are then zero and we neglect them writing

$$
y_{j}=\ell\left(\sum_{n=0}^{j-1} \cos \left(\sum_{i=1}^{n} \beta_{i}-\varphi\right), \sum_{n=0}^{j-1} \sin \left(\sum_{i=1}^{n} \beta_{i}-\varphi\right)\right),
$$

where $\varphi \in \mathbb{R}$ is a free parameter and $\beta_{i}$ is the "bending angle" at the $i$ th vertex (modulo $2 \pi$ ); the family of these angles satisfies naturally the condition

$$
\sum_{i=1}^{N} \beta_{i}=2 \pi w
$$

for some $w \in \mathbb{Z}$. Choosing $\tilde{\varphi}=\frac{\pi}{N}$ and $\tilde{\beta}_{i}=\frac{2 \pi i}{N}$, we get in particular

$$
\tilde{y}_{ \pm m}=\ell\left( \pm \sum_{n=0}^{m-1} \cos \frac{\pi}{N}(2 n+1), \sum_{n=0}^{m-1} \sin \frac{\pi}{N}(2 n+1)\right) \text {. }
$$

Then we have

$$
\left|\tilde{y}_{j}-\tilde{y}_{j \pm m}\right|=\ell\left[\left(\sum_{n=0}^{m-1} \cos \frac{\pi}{N}(2 n+1)\right)^{2}+\left(\sum_{n=0}^{m-1} \sin \frac{\pi}{N}(2 n+1)\right)^{2}\right]=: \ell \Upsilon_{m}
$$

and consequently, $\nabla_{j} K_{m}\left(\tilde{y}_{1}, \ldots, \tilde{y}_{N}\right)=0$ holds for $j=1, \ldots, N$ if we choose all the Lagrange multipliers in (4.1) equal to

$$
\lambda=\frac{\sigma_{m}}{N \Upsilon_{m}} \quad \text { with } \quad \sigma_{m}:=\frac{\sum_{n=0}^{m-1} \sin \frac{\pi}{N}(2 n+1)}{\sin \frac{\pi}{N}}=\frac{\sin ^{2} \frac{\pi m}{N}}{\sin ^{2} \frac{\pi}{N}} .
$$

The second partial derivatives, $\nabla_{k, r} \nabla_{j, s} K_{m}\left(y_{1}, \ldots, y_{N}\right)$, are computed to be

$$
\begin{aligned}
\frac{1}{N}\{ & \frac{\delta_{k j}-\delta_{k, j+m}}{\left|y_{j}-y_{j+m}\right|} \delta_{r s}-\frac{\left(y_{j}-y_{j+m}\right)_{r}\left(y_{j}-y_{j+m}\right)_{s}\left(\delta_{k j}-\delta_{k, j+m}\right)}{\left|y_{j}-y_{j+m}\right|^{3}}+\frac{\delta_{k j}-\delta_{k, j-m}}{\left|y_{j}-y_{j-m}\right|} \delta_{r s} \\
& \left.-\frac{\left(y_{j}-y_{j-m}\right)_{r}\left(y_{j}-y_{j-m}\right)_{s}\left(\delta_{k j}-\delta_{k, j-m}\right)}{\left|y_{j}-y_{j-m}\right|^{3}}+\frac{\lambda}{\ell}\left(\delta_{k, j+m}+\delta_{k, j-m}-2 \delta_{k j}\right) \delta_{r s}\right\}
\end{aligned}
$$


This allows us to evaluate the Hessian at the stationary point. After a long but straightforward calculation we arrive at the expression

$$
\begin{aligned}
& \sum_{k, j, r, s} \nabla_{k, r} \nabla_{j, s} K_{m}\left(\tilde{y}_{1}, \ldots, \tilde{y}_{N}\right) \xi_{k, r} \xi_{j, s} \\
& \quad=\frac{1}{N \ell \Upsilon_{m}} \sum_{j=1}^{N}\left\{\left|\xi_{j}-\xi_{j+m}\right|^{2}-\frac{\left(\xi_{j}-\xi_{j+m}, \tilde{y}_{j}-\tilde{y}_{j+m}\right)^{2}}{\left|\tilde{y}_{j}-\tilde{y}_{j+m}\right|^{2}}-\sigma_{m}\left|\xi_{j}-\xi_{j+1}\right|^{2}\right\} .
\end{aligned}
$$

We observe that the form depends on vector differences only, so it is invariant with respect to Euclidean transformations. Furthermore, the sum of the first two terms in the bracket at the r.h.s. of (4.5) is non-negative by Schwarz inequality.

Since the second term in non-positive, it will be sufficient to establish negative definiteness of the quadratic form

$$
\xi \mapsto S_{m}[\xi]:=\sum_{j}\left\{\left|\xi_{j}-\xi_{j+m}\right|^{2}-\sigma_{m}\left|\xi_{j}-\xi_{j+1}\right|^{2}\right\}
$$

on $\mathbb{R}^{N d}$. Moreover, it is enough to consider here the case $d=1$ only because $S_{m}$ is a sum of its "component" forms. We observe that the matrices corresponding to the two parts of (4.6) can be simultaneously diagonalized; the corresponding eigenfunctions are $\left\{\left(\begin{array}{c}\sin \\ \cos \end{array}\right)\left(\mu_{r} j\right)\right\}_{j=1}^{N}$, where $\mu_{r}=\frac{2 \pi r}{N}, r=0,1, \ldots, m-1$. Taking the corresponding eigenvalues we see that it is necessary to establish the inequalities

$$
4\left\{\sin ^{2} \frac{\pi m r}{N}-\sigma_{m} \sin ^{2} \frac{\pi r}{N}\right\}<0
$$

for $m=2, \ldots,\left[\frac{1}{2} N\right]$ and $r=2, \ldots, m-1$. We left out here the case $r=1$ when the l.h.s. of (4.7) vanishes. At the same time, however, the above explicit form of the eigenfunctions shows that the corresponding $\xi_{j}-\xi_{j+m}$ are in this case proportional to $\tilde{y}_{j}-\tilde{y}_{j+m}$ so the second term at the r.h.s. of (4.5) is negative unless $\xi=0$.

Using the expression (4.4) for $\sigma_{m}$ we can rewrite the condition (4.7) in terms of Chebyshev polynomials of the second kind as

$$
U_{m-1}\left(\cos \frac{\pi}{N}\right)>\left|U_{m-1}\left(\cos \frac{\pi r}{N}\right)\right|,
$$

which can be checked using properties of these polynomials [AS, Chap. 22]. One can do it also directly, because (4.8) is equivalent to

$$
\sin \frac{\pi m}{N} \sin \frac{\pi r}{N}>\left|\sin \frac{\pi}{N} \sin \frac{\pi m r}{N}\right|, \quad 2 \leq r<m \leq\left[\frac{N}{2}\right] .
$$

We have $\sin x \sin \left(\eta^{2} / x\right) \geq \sin \eta$ for a fixed $\eta \in\left(0, \frac{1}{2} \pi\right)$ and $2 \eta^{2} / \pi \leq x \leq \frac{1}{2} \pi$, and moreover, this inequality is sharp if $x \neq \eta$, hence the desired assertion follows from the inequality $\sin ^{2} x-\sin \frac{\pi}{N} \sin \frac{N x^{2}}{\pi} \geq 0$ valid for $x \in\left(0, \frac{1}{2} \pi\right)$. This concludes the proof of Theorem 4.1, and by that also of Theorem 2.1 


\section{Global properties}

The question whether the maximizer represented by regular polygons is global at the same time is more difficult. By the argument of Sec. 3 it can be reduced again to a purely geometric problem, namely that about validity of the following claim.

Conjecture 5.1 The property $\left(P_{m}\right)$ holds globally for any $m=2, \ldots,\left[\frac{1}{2} N\right]$.

Let us look at the problem in more detail in the particular case of planar polygons, $d=2$. We employ a parametrization analogous to (4.2): for a fixed $i$ we identify $y_{i}$ with the origin and set for simplicity $\varphi=0$, i.e.

$$
y_{i+m}=\ell\left(1+\sum_{n=1}^{m-1} \cos \sum_{j=1}^{n} \beta_{j+i}, \sum_{n=1}^{m-1} \sin \sum_{j=1}^{n} \beta_{j+i}\right)
$$

in addition to the angular condition (4.3) we require naturally also that $y_{i}=y_{i+N}$, or in other words

$$
1+\sum_{n=1}^{N-1} \cos \sum_{j=1}^{n} \beta_{j+i}=\sum_{n=1}^{N-1} \sin \sum_{j=1}^{n} \beta_{j+i}=0
$$

for any $i=1, \ldots, N$. The mean length of all $m$-diagonals is easily found,

$$
M_{m}=\frac{\ell}{N} \sum_{i=1}^{N}\left[\left(1+\sum_{n=1}^{m-1} \cos \sum_{j=1}^{n} \beta_{j+i}\right)^{2}+\left(\sum_{n=1}^{m-1} \sin \sum_{j=1}^{n} \beta_{j+i}\right)^{2}\right]^{1 / 2}
$$

or alternatively

$$
M_{m}=\frac{\ell}{N} \sum_{i=1}^{N}\left[m+2 \sum_{n=1}^{m-1} \sum_{r=1}^{n} \cos \sum_{j=r}^{n} \beta_{j+i}\right]^{1 / 2} .
$$

It allows us to prove the claim in the simplest nontrivial case.

Proposition 5.2 The property $\left(P_{2}\right)$ holds globally if $d=2$. 
Proof: By (5.2) the mean length of the 2-diagonals equals

$$
M_{2}=\frac{\sqrt{2} \ell}{N} \sum_{i=1}^{N}\left(1+\cos \beta_{i}\right)^{1 / 2}=\frac{2 \ell}{N} \sum_{i=1}^{N} \cos \frac{\beta_{i}}{2} ;
$$

notice that $\cos \frac{\beta_{i}}{2}>0$ because $\beta_{i} \in(-\pi, \pi)$. Using now convexity of the function $u \mapsto-\cos \frac{u}{2}$ in $(-\pi, \pi)$ together with the condition (4.3) we find

$$
-\sum_{i=1}^{N} \cos \frac{\beta_{i}}{2} \geq-N \cos \left(\sum_{i=1}^{N} \frac{\beta_{i}}{2}\right)=-N \cos \frac{\pi}{N},
$$

and therefore $M_{2} \leq 2 \ell \cos \frac{\pi}{N}=\tilde{M}_{2}$. Moreover, since the said function is strictly convex, the inequality is sharp unless all the $\beta_{i}$ 's are the same.

For $m \geq 3$ the situation is more complicated and one has to take into account also the condition (5.1); for the moment the problems remains open.

\section{Possible extensions}

Apart of proving Conjecture 5.1 and by that the global uniqueness of the maximizer, the present problem offers various other extensions. One can ask, for instance, what will be the maximizer when we replace the equilaterality by a prescribed ordered $N$-tuple of polygon lengths $\left\{\ell_{j}\right\}$ and/or coupling constants $\left\{\alpha_{j}\right\}$. In both cases the task becomes more difficult because we loose the ground state symmetry which yielded the relation (3.3) and consequently the geometric reformulation based on the inequality (3.4).

One can also attempt to extend the result to point interaction family of point interactions in $\mathbb{R}^{3}$ placed on a closed surface. In this case, however, there is no unique counterpart to the equilaterality and one has to decide first what the "basic cell" of such a polyhedron surface should be. Another extensions of our isoperimetric problem concern "continuous" versions of the present situation, i.e. Schrödinger operators with singular interactions supported by closed curves or surfaces - cf. [EI, Ex2] and references therein - or with a regular potential well extended along a closed curve.

\section{Acknowledgments}

The research has been partially supported by ASCR within the project K1010104. 


\section{References}

[AS] M.S. Abramowitz, I.A. Stegun, eds.: Handbook of Mathematical Functions, Dover, New York 1965.

[AGHH] S. Albeverio, F. Gesztesy, R. Høegh-Krohn, H. Holden: Solvable Models in Quantum Mechanics, 2nd printing, AMS, Providence, R.I., 2004.

[AB] M.S. Ashbaugh, R.D. Benguria: A sharp bound for the ratio of the first two eigenvalues of Dirichlet Laplacians and extensions, Ann. Math. 135 (1992), 601-628.

[Ex1] P. Exner: Bound states of infinite curved polymer chains, Lett. Math. Phys. 57 (2001), 87-96.

[Ex2] P. Exner: Spectral properties of Schrödinger operators with a strongly attractive $\delta$ interaction supported by a surface, in AMS "Contemporary Mathematics" Series, vol. 339, Providence, R.I., 2003; pp. 25-36.

[EHL] P. Exner, E.M. Harrell, M. Loss: Optimal eigenvalues for some Laplacians and Schrödinger operators depending on curvature, in Operator Theory : Advances and Applications, vol. 108; Birkhäuser Verlag, Basel 1998; pp. 47-53.

[EI] P. Exner, T. Ichinose: Geometrically induced spectrum in curved leaky wires, J. Phys. A: Math. Gen. 34 (2001), 1439-1450.

[EN] P. Exner, K. Němcová: Leaky quantum graphs: approximations by point interaction Hamiltonians, J. Phys. A: Math. Gen. 36 (2003), 10173-10193.

[Fa] G. Faber: Beweiss das unter allen homogenen Membranen von Gleicher Fläche und gleicher Spannung die kreisförmige den Tiefsten Grundton gibt, Sitzungber. der math.-phys. Klasse der Bayerische Akad. der Wiss. zu München (1923), 169-172.

[Kr] E. Krahn: Über eine von Rayleigh formulierte minimal Eigenschaft des Kreises, Ann. Math. 94 (1925), 97-100.

[KL] M.G. Krein, G.K. Langer: On deficiency subspaces of a Hermitean operator, Sov. J. Funct. Anal. Appl. 5 (1971), 59-71. 